TRANSACTIONS OF THE

AMERICAN MATHEMATICAL SOCIETY

Volume 225, 1977

\title{
ON SUBCATEGORIES OF TOP
}

BY

\author{
S. P. FRANKLIN, D. J. LUTZER AND B. V. S. THOMAS
}

\begin{abstract}
A categorical characterization of a subcategory $S$ of TOP (or $T_{2}$ ) is one which enables the identification of $S$ in TOP (or $T_{2}$ ) without requiring the reconstruction of the topological structure of its objects. In this paper we so characterize various familiar subcategories of TOP (Hausdorff spaces, normal spaces, compact Hausdorff spaces, paracompact Hausdorff spaces, metrizable spaces, first countable spaces) in terms of the global behavior of the (objects and) morphisms of the subcategory.
\end{abstract}

0. Introduction. A categorical characterization of a subcategory $S$ of TOP (or of $T_{2}$ ) is one which enables the identification of $S$ in TOP (or $T_{2}$ ) without requiring the reconstruction of the topological structure of its objects. The proof of the triviality of the automorphism class group of TOP [Fe, p. 32] assures us of the existence of such characterizations. As will be shown in $\S 1$, this proof establishes that all the topological structure of an object of TOP can be recovered from the behavior of the arrows with that object as source and/ or target. In $\$ 2$ we establish a number of lemmas (mostly known), and we use these lemmas in $\$ 3$ to characterize many important subcategories of TOP and/ or $T_{2}$, such as the metrizable spaces, the paracompact Hausdorff spaces, the normal spaces, etc.

We can distinguish at least three classes of "categorical" characterizations of subcategories of TOP, which are listed below:

Class 3. Straightforward translations of the familiar definitions. These can always be obtained because of the results in $\S 1$. Example: Characterize the concepts "open", "cover", "refinement", "locally finite" categorically and then the standard definition of paracompact spaces is categorical.

Class 2. Characterizations using standard categorical constructions (product, coproduct, epimorphic images, etc.) but in terms of individual objects, and/or constants outside the subcategory. Example: The category of paracompact Hausdorff spaces contains precisely those Hausdorff spaces $X$ such that

Received by the editors March 6, 1974 and, in revised form, February 24, 1975 and September 16, 1975.

AMS (MOS) subject classifications (1970). Primary 54A99, 54H99; Secondary 54C10, 54D10, 54D15, 54D20, 54D30, 54D45, 54E35.

Key words and phrases. Categorical topology, categorical characterizations. 
$X \times \beta X$ is normal (presuming we can give Class 2 definitions of normality and of $\beta X$ ).

Class 1. Characterizations in terms of the global behavior of the (objects and) morphisms of the subcategory, preferably without the use of constants, and certainly without the use of constants outside the subcategory. Example: The paracompact Hausdorff spaces form the largest left-fitting subcategory of $T_{2}$ preserved by shrinks of $T_{2}$ extremal monos.

Our aim in this paper is to characterize categorically various familiar subcategories of TOP and $T_{2}$, preferably with Class 1 characterizations.

1. The arrows of TOP determine the spaces and functions. Assume that we are given a collection of dots and arrows and are told that it is the category TOP. We can reconstruct the topological spaces and the functions as follows. See [Fe, p. 32].

1.1. The singleton space, $*$, is the terminal object of TOP. We can now recover the underlying set of an object $A$ of TOP; it is precisely TOP $(*, A)$. Then, given an arrow $f: A \rightarrow B$ in TOP, and an element $a \in A$ (i.e., an arrow $a: * \rightarrow A), f(a)$ is the composition $f \circ a: * \rightarrow B$.

1.2. The Sierpin'ski two point space $S$, i.e., the two element space with only one isolated point, is the only object, up to isomorphism, with precisely three self-maps. Any space with three or more points has at least four self-maps: each constant and the identity map; * has one self-map. The two point discrete and indiscrete spaces each have four self-maps; hence, only $S$ has three selfmaps.

1.3. There are two arrows $* \rightarrow S$. We wish to characterize the open map, $u: * \rightarrow S$, and the closed map, c: $* \rightarrow S$. Let $f: * \rightarrow S$ be one of these arrows, and let $A$ be an object of TOP. For each $g: A \rightarrow S$ let $A_{g}$ be the set of all arrows $a: * \rightarrow A$ such that the following triangle commutes.

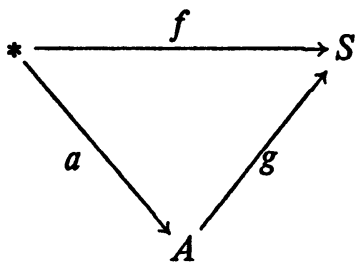

We say $A$ is a test space for $f$ if for all $a: * \rightarrow A$, there is a morphism $g: A \rightarrow S$ such that $A_{g}=\{a\}$. (Notice that if $f=c$, the test spaces are the $T_{1}$ spaces; and if $f=u$ the test spaces are the discrete spaces.) Then $f=u$ if and only if for every test space $A$ and each $\mathscr{C} \subset$ TOP $(*, A)$, there exists a $g: A \rightarrow S$ such that $\mathbb{Q}=A_{g}$. (This says nothing more than that if points are open then every subset is open, but there are $T_{1}$ spaces with nonclosed subsets.) Let 0 denote $u(*)$, and 1 denote $c(*)$. 
We now wish to determine the closed and the open subsets of an object $A$ of TOP. We need two standard definitions.

1.4. Definition. A morphism $e^{\#}: A \rightarrow B$ in a category $\mathcal{C}$ is an extremal epimorphism if $e^{\#}$ is an epimorphism such that whenever $e^{\#}=m r$

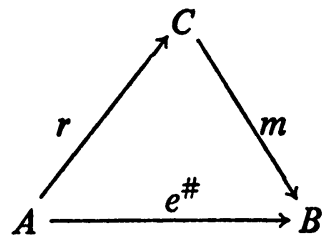

for any morphism $r: A \rightarrow C$ and any monomorphism $m: C \rightarrow B$, then $m$ is an isomorphism. Dually, a morphism $m^{\#}: B \rightarrow A$ in a category $C$ is an extremal monomorphism if $m^{\#}$ is a monomorphism such that whenever $m^{\#}=l e$

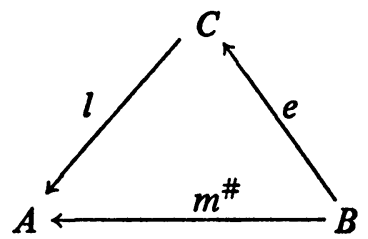

for any morphism $l: C \rightarrow A$ and any epimorphism $e: B \rightarrow C$, then $e$ is an isomorphism.

1.5. Notation. $\rightarrow$ will denote an arbitrary morphism,

$\rightarrow$ will denote an epimorphism,

$\rightarrow \rightarrow$ will denote an extremal epi,

$\longleftrightarrow$ will denote a monomorphism,

$\longleftrightarrow$ will denote an extremal mono.

The following three results are in the literature $[\mathbf{H}, \mathrm{pp} .115,116]$ and $[\mathbf{F r} 3$, pp. 22-24].

1.6. A subspace $F \stackrel{m^{*}}{\longrightarrow} A$ of an object $A$ of $\mathrm{TOP}$ is an object $F$ of TOP together with a TOP extremal monomorphism; or, the extremal monomorphisms in TOP are precisely the embeddings.

1.7. The extremal monomorphisms in $T_{2}$ are precisely the closed embeddings.

1.8. In TOP and $T_{2}$ the extremal epimorphisms are the quotient maps.

We will need the following purely categorical lemma.

1.9. LemMa. If the composition $B \stackrel{f}{\rightarrow} A \stackrel{g}{\rightarrow} A^{\prime}$ is an extremal monomorphism, then $f$ is an extremal monomorphism.

Proof. It is well known that if $g f$ is a monomorphism, then $f$ is a monomorphism. Suppose $f=l e$ with $e$ an epimorphism; then the following diagram commutes: 


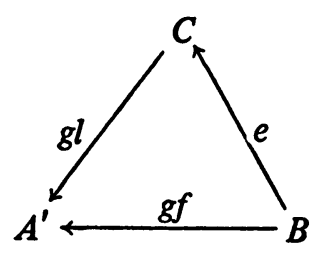

Hence $e$ is an isomorphism.

We are now in a position to recover the topology of an object of TOP.

1.10. LEMMA. A subspace $F \stackrel{m^{*}}{\longrightarrow} A$ of an object $A$ in TOP is closed if and only if there is some $f: A \rightarrow S$ such that

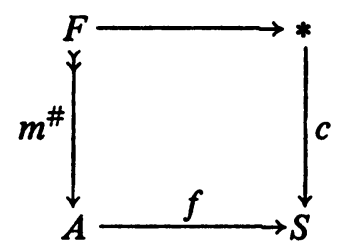

is a pullback diagram.

Proof. Suppose $F$ is a closed subspace of $A$ and let $f: A \rightarrow S$ be the (continuous) characteristic function of $F(1$ is the closed point of $S$ ). Then the diagram

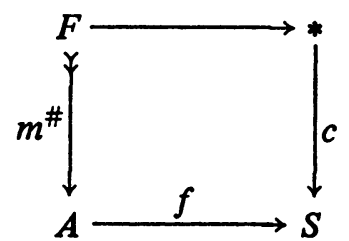

commutes, and is the desired pullback.

Conversely, suppose there is an $f: A \rightarrow S$ such that

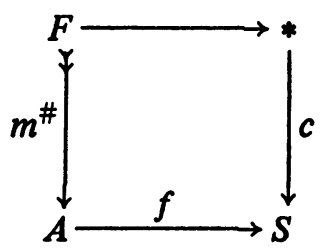

is a pullback. Since $A \stackrel{i}{\leftarrow} f^{\leftarrow}(1) \rightarrow *$ is also a pullback for $A \stackrel{f}{\rightarrow} S \leftarrow$ * , there must be an isomorphism $g: f^{\leftarrow}(1) \rightarrow F$ such that $m^{\#} g=i$.

1.11. LEMMA. $A$ subspace $G \stackrel{m^{*}}{\longrightarrow} A$ of an object $A$ of TOP is open if and only if there is some $g: A \rightarrow S$ such that 


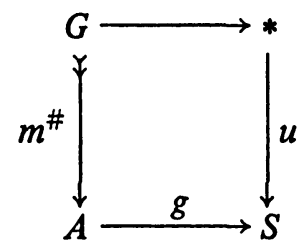

is a pullback diagram.

The proof is similar to the proof of Lemma 1.10 with $g=\chi_{A \backslash G}$.

These last two lemmas have the disadvantage that they will have no meaning in categories which do not contain a Sierpiński two point space, e.g., $T_{2}$. This same disadvantage is shared by the characterizations of closed maps and open maps in TOP (Lemmas 2.2 and 2.3).

2. More definitions and lemmas. We have shown in $\$ 1$ that the topology of an object of TOP can be recovered from the behavior of the arrows with that object as source and/or target. In this section we establish a number of lemmas which will be used in the characterizations in \$3. The impatient reader may skip this section if he is willing to assume that we can establish categorical meanings for the topological terms used in the third section.

The following lemma appears in [H, p. 65] and in [Fr3, p. 33].

2.1. LEMMA. In any category with the extremal epi-mono factorization property,

(i) factorization is unique and extremal epimorphisms compose if and only if

(ii) for each commutative diagram

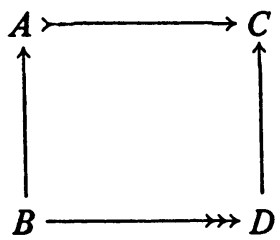

there exists a unique $d: D \rightarrow A$ making everything commute.

In particular, TOP has these properties and their duals.

2.2. LEMMA. $A$ TOP-morphism $h: A \rightarrow B$ is closed if and only if for each closed $m^{\#}: F \ggg A, h m^{\#}$ can be factored $h m^{\#}=n^{\#} v$ where $n^{\#}: F^{\prime} \ggg B$ is closed and $v: F \rightarrow F^{\prime}$ is epi.

Proof. Consider the following diagram.

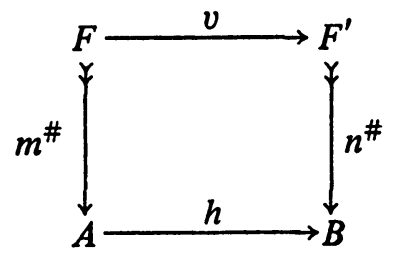


Using 2.1 it is clear that $F^{\prime}$ is, in fact, $h(F)$ and that the lemma is merely a categorical restatement of the definition of a closed map.

2.3. LEMMA. $A$ TOP-morphism $h: A \rightarrow B$ is open if and only if for each open $m^{\#}: G \gg A, h m^{\#}$ can be factored $h m^{\#}=n^{\#} v$ where $n^{\#}: F^{\prime} \gg B$ is open and $v: F \rightarrow F^{\prime}$ is epi.

We can now characterize perfect maps in terms of closed maps. Notice that for us a perfect map need not be onto.

2.4. LemMa. $A$ TOP-morphism $f: A \rightarrow B$ is perfect if and only if $f \times 1_{Z}: A \times Z$ $\rightarrow B \times Z$ is closed for every identity ${ }_{Z}: Z \rightarrow Z$.

Proof. [Bo, p. 117].

If we restrict our attention to $T_{2}$ we can describe closed maps and perfect maps without relying on constants.

2.5. LeMMA. In $T_{2}$ a morphism $f: X \rightarrow Y$ is a closed map if and only if for every extremal mono $m^{\#}: E \nrightarrow X, f m^{\#}$ has an external epi-extremal mono factorization.

Proof. Let $f: X \rightarrow Y$ be closed and $m^{\#}: E \rightarrow X$ be an extremal mono. Then $f(E)$ is a closed subset of $Y$ and $\left.f\right|_{E}: E \rightarrow f(E)$ is a closed map. Thus

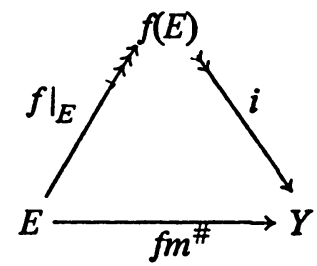

is the desired factorization.

Conversely, suppose $f$ has the factorization property and $E$ is a closed subset of $X$. Let $m^{\#}$ be the inclusion map $E \ggg X$. Then there is a factorization

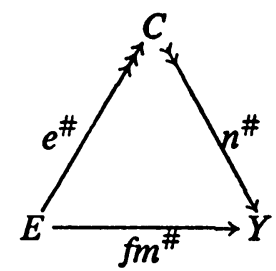

It follows that $f(E)=f m^{\#}(E)=n^{\#}(C)$ which is a closed subset of $Y$.

Thus, using Lemma 2.4, we have a characterization of the perfect maps in $T_{2}$ which does not use constants. In what follows we will have occasion to use the fact that a perfect epi is necessarily onto. 
Now that we have characterized perfect-onto maps categorically, the following definition is also categorical.

2.6. Definition. A subcategory $e$ of TOP (or $T_{2}$ ) is called right-fitting if perfect-onto images of objects in $\mathcal{C}$ are again in $\mathcal{C} C$ is called left-fitting if perfect-onto preimages of objects of $\mathcal{C}$ are again in $\mathcal{C} C$ is called fitting if it is both left-fitting and right-fitting.

We close this section with a categorical characterization of biquotient maps in the style of 2.4 .

2.7. Lemma. $A$ morphisn $f: A \rightarrow B$ in $T_{2}$ is biquotient if and only if $f \times 1_{C}$ : $A \times C \rightarrow B \times C$ is an extremal epimorphism for every $1_{C}: C \rightarrow C$ in $T_{2}$.

Proof. [M, p. 288].

3. Characterizations. Now that we have shown what is meant categorically by such terms as "subspace", "closed map", etc., we will freely use such common terms as "hereditary", "preserved by closed maps", etc. The word "subcategory" will always denote a full and replete subcategory. For later theorems it will be convenient to have categorical characterizations of $T_{2}$ and $T_{4}$; for completeness we include categorical characterizations of the major separation axioms.

3.1. Proposition. $T_{0}$ is the epireflective hull of the Sierpin'ski two point space.

Proof. This proposition is a restatement of Alexandroff's result that every $T_{0}$ space is a subspace of a product of Sierpiniski spaces [A] using the fact that epireflective hulls of TOP are produced by taking subspaces of products $[\mathbf{H}]$, [Fr3].

3.2. PROPOSIrION. $T_{1}$ is the largest epireflective subcategory of TOP which is properly contained in $T_{0}$.

Proof. Since $T_{1}$ is hereditary and productive, it is epireflective in TOP [H],

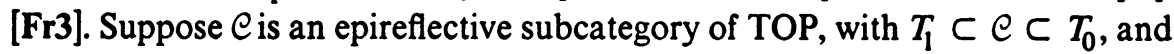
suppose that there is a space $X \in \mathrm{Ob} \backslash \backslash \mathrm{Ob} T_{1}$. Then $S$ is a subspace of $X$ and, since $\mathcal{C}$ is epireflective, $T_{0} \subset \mathcal{C}$.

3.3. PROPOSITION. $T_{2}$ is the largest subcategory of TOP such that equalizers are precisely the closed TOP extremal monos.

Proof. It is well known that if

$$
X \underset{\mathrm{g}}{\stackrel{f}{\Rightarrow}} Y
$$

in $T_{2}$, then the equalizer of $f$ and $g,\{x \in X \mid f(x)=g(x)\}$, is a closed subspace of $X$. Conversely, if $F$ is a closed subspace of $X \in \mathrm{Ob} T_{2}$, then $F$ is the 
equalizer of the maps

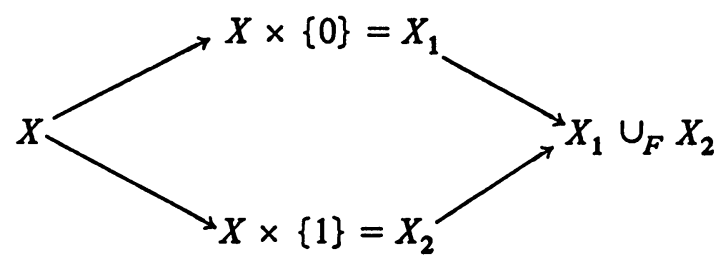

and, since $F$ is closed, $X_{1} \cup_{F} X_{2}$ is indeed a Hausdorff space.

On the other hand, suppose $C$ is any subcategory of TOP properly containing $T_{2}$ and that $X \in \mathrm{Ob} \backslash \backslash \mathrm{Ob} T_{2}$. Then there is a net $S: D \rightarrow X$ which converges to two distinct points, say $x_{1}$ and $x_{2}$, of $X$. Without loss of generality we may assume that $D$ has no largest element. Then the net space $D^{*}$ $=D \cup\{\infty\}$ (the tails of $D$ determine a neighborhood basis at $\infty$ ) and its discrete subspace $D$ are Hausdorff, and hence belong to $C$. For $i=1,2$ the maps $S_{i}: D^{*} \rightarrow X$ defined by

$$
S_{i}(d)= \begin{cases}x_{i} & \text { if } d=\infty \\ S(d) & \text { otherwise }\end{cases}
$$

are continuous, since $S$ converges to both $x_{1}$ and $x_{2}$. Their equalizer $D$ is not closed in $D^{*}$.

It would be particularly pleasing to find a characterization of $T_{2}$ which avoids identifying the closed subspaces, since $T_{2}$ is such an important subcategory of TOP.

3.4. Proposition. $T_{3}$ is the smallest nontrivial, fitting, hereditary subcategory of $T_{2}$.

It is clear that $T_{3}$ is hereditary and right-fitting in $T_{2}$. To see that $T_{3}$ is leftfitting let $p: X \rightarrow Y$ be a perfect map with $Y \in \mathrm{Ob} T_{3}$ and $X \in \mathrm{Ob} T_{2}$. Let $F$ be a closed set in $X$ and let $x$ be a point of $X$ not in $F$. If $p(x) \notin p(F)$ there is no problem separating $x$ from $F$, so suppose $p(x) \in p(F)$. This means that $p^{\leftarrow}[p(x)] \cap F \neq \varnothing$. Since $X$ is Hausdorff the compact set $p^{\leftarrow}[p(x)] \cap F$ and the point $x$ can be separated by disjoint open sets $U_{1}$ and $V_{1}$, say $x \in U_{1}$ and $p^{\leftarrow}[p(x)] \cap F \subset V_{1}$. Thus $p^{\leftarrow}[p(x)] \cap\left(F \backslash V_{1}\right)=\varnothing$ and $p(x) \notin p\left(F \backslash V_{1}\right)$. Hence $x$ and $F \backslash V_{1}$ can be separated by disjoint open sets $U_{2}$ and $V_{2}$, say $x \in U_{2}, F \backslash V_{1} \subset V_{2}$. Then $U_{1} \cap U_{2}$ and $V_{1} \cup V_{2}$ are open sets separating $x$ from $F$.

Let $C$ be a subcategory of $T_{2}$ satisfying the above hypotheses. Since $C$ is nontrivial and hereditary it contains $*$; since $\mathcal{C}$ is left-fitting it contains all compact Hausdorff spaces. The hypothesis that $\mathcal{C}$ is hereditary now puts all Tychonoff spaces in $\mathcal{C}$; and since $C$ is right-fitting it contains $T_{3}$, since every 
regular space is the perfect image of its projective cover which is Tychonoff (see $[\mathrm{Ba}]$ ).

The standard result that $T_{31 / 2}$ is the hereditary hull of the category of compact Hausdorff spaces will be meaningful in this context once a categorical characterization has been given for $\mathrm{CH}$; see Propositions 3.8, 3.9, and 3.10.

The following definition is needed for the characterization of $T_{4}$.

3.5. Definition. A morphism $f: X \rightarrow Y$ is a shrink of $m: F \rightarrow X$ if there is a $g: * \rightarrow Y$ such that

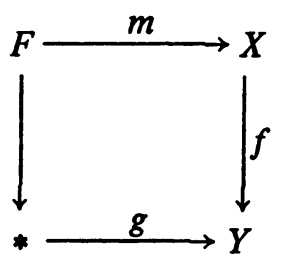

is a pushout.

3.6. Proposition. $T_{4}$ is the largest subcategory of $T_{2}$ preserved by shrinks, in TOP, of $T_{2}$ extremal monos.

3.7. LEMMA. In TOP, a shrink of an extremal mono $m^{\#}: F \gg X$ is the quotient map which collapses $F$ to a point.

Proof. The quotient map $q: X \rightarrow X / F$ has the pushout property, and pushouts are unique.

Proof of Proposition 3.6. Since a shrink of a $T_{2}$ extremal mono is a closed map, $T_{4}$ is preserved by them. Suppose that $C$ is a subcategory of $T_{2}$ preserved by shrinks of $T_{2}$ extremal monos, and let $F_{1}$ and $F_{2}$ be disjoint closed subsets of a space $X$ in $C$. First shrink $F_{1}$ to a point, and then shrink $F_{2}$ to a point. Since the resulting space must be Hausdorff, $X$ itself must be normal. Thus any such $C$ can contain only normal spaces, and $T_{4}$ is the largest such subcategory.

We give three characterizations of the subcategory $\mathrm{CH}$ of compact Hausdorff spaces.

3.8. THEOREM (HERRLICH AND STRECKER). $\mathrm{CH}$ is the only nontrivial, epireflective subcategory of $T_{2}$ which is varietal.

This theorem is proved in [H-S].

3.9. THEOREM (DE GROOT). $\mathrm{CH}$ is the only nontrivial, productive subcategory $C_{\text {of TOP }}$ which is preserved by closed epis and satisfies

$$
\begin{aligned}
& \text { if } X \in \mathrm{ObC} \text { and } m^{\#}: F \rightsquigarrow X, \text { then } \\
& F \in \mathrm{Ob} C \text { if and only if } m^{\#} \text { is a closed map. }
\end{aligned}
$$

Proof. In [W, p. 51] there is a topological version of this result, due to de 
Groot, which needs only to be translated into categorical language to yield 3.9 [F-T].

3.10. Proposinion. $\mathrm{CH}$ is the only nontrivial, productive, left-fitting subcategory of $T_{2}$ preserved by shrinks, in TOP, of $T_{2}$ extremal monos.

Proof. If $C$ is a subcategory of $T_{2}$ satisfying these conditions, then $C$ contains some nonempty space $X$. Since $\mathcal{C}$ is left-fitting, * $\in \mathrm{Ob} \mathcal{C}$ and, hence, $\mathrm{CH} \subset \mathcal{C}$. On the other hand $C \subset T_{4}$ since $T_{4}$ is the largest subcategory preserved by shrinks of $T_{2}$ extremal monos. Thus for any $X \in \mathrm{Ob} \mathcal{C}, X^{\kappa} \in \mathrm{Ob} \mathcal{C} \subset \mathrm{Ob} T_{4}$ for any cardinal $\kappa$, and it follows from Noble's theorem [N] that $X$ is a compact Hausdorff space.

3.11. Proposition. The paracompact spaces form the largest left-fitting subcategory of $T_{2}$ which is preserved by shrinks, in TOP, of $T_{2}$ extremal monos.

Proof. Since shrinks of $T_{2}$ extremal monos are closed maps, the category of paracompact spaces clearly satisfies the conditions of the theorem.

Let $\mathrm{C}$ be a subcategory of $T_{2}$ satisfying these conditions; then $\mathrm{CH} \subset \mathcal{C} \subset T_{4}$ by previous arguments (Propositions 3.6 and 3.10). Furthermore, since $e$ is left-fitting, for each $X \in \mathrm{Ob} \mathcal{C}, X \times \beta X \in \mathrm{Ob} \mathcal{C}$ (see [Fr2]), and thus by the theorem of Tamano and Morita [T], [Mo], $X$ is paracompact.

3.12. Proposition. The locally compact Hausdorff spaces form the smallest nontrivial, coproductive, left-fitting subcategory of $T_{2}$ preserved by biquotient maps (in $T_{2}$ ).

Proof. Let $C$ be a category satisfying the above conditions. Since $C$ is leftfitting and nontrivial, $\mathrm{CH} \subset \mathrm{C}$. Let $X$ be any locally compact Hausdorff space and let $\left\{K_{\alpha}\right\}_{\alpha \in A}$ be all the compact subsets of $X$; then the disjoint union of the $K_{\alpha}$ 's, $\cup_{\alpha} K_{\alpha}$ is an object of $\mathcal{C}$. The natural quotient map $\cup_{\alpha} K_{\alpha} \rightarrow X$ is, in fact, biquotient, and hence $X \in \mathrm{Ob}$. Since the locally compact Hausdorff spaces satisfy the condition of the proposition, they form the smallest such category.

3.13. Proposition. The separable Hausdorff spaces form the smallest nontrivial, countably coproductive subcategory of $T_{2}$ preserved by epis (in $T_{2}$ ).

Proof. Every separable Hausdorff space is the codomain of some epimorphism (in $T_{2}$ ) with domain $\mathbf{N}$, which is the countable coproduct of singleton spaces.

It is interesting to note the (superficially) minor differences in the next three characterizations.

3.14. PROPOSITION. The metrizable spaces form the smallest nontrivial subcategory of $T_{2}$ which is

(1) countably productive, 
(2) hereditary,

(3) coproductive,

(4) right-fitting (in $T_{2}$ ).

Proof. Franklin has shown [Fr1] that any metric space is the perfect image of a subspace of a countable product of discrete spaces.

3.15. Proposition. The separable metrizable spaces form the smallest nontrivial subcategory of $T_{2}$ which is

(1) countably productive,

(2) hereditary,

(3) countably coproductive,

(4) right-fitting (in $T_{2}$ ).

Proof. If $\mathcal{C}$ is any category satisfying the stated conditions, it contains $*$, by (2) and, hence by (3), it contains the two point discrete space. Use of (1) puts the Cantor set in $C$, and (4) yields the unit interval. Another application of (1) gives us the Hilbert cube, and (2) all the separable metric spaces.

Another proof can be given along the lines of the previous proposition, i.e., we could show more directly that every separable metric space is the perfect image of a subspace of a countable product of countable discrete spaces.

3.16. Proposition. The first countable spaces form the smallest nontrivial subcategory of TOP which is

(1) countably productive,

(2) hereditary,

(3) coproductive,

(4) preserved by open TOP epis.

Proof. As we have seen in previous proofs, any such category must contain all discrete spaces. Ponomarev has shown that every first countable $T_{1}$ space is the open image of a subspace of a countable product of discrete spaces [P]. Michael has since shown that any first countable space, $T_{1}$ or not, is the open image of a first countable Hausdorff space [M2].

\section{BIBLIOGRAPHY}

[A] P. Alexandroff, Zur Theorie der topologischen Räume, C. R. (Dokl.) Acad. Sci. USSR 2 (1936), 55-58.

[Ba] B. Banaschewski, Projective covers in categories of topological spaces and topological algebras, General Topology and its Relations to Modern Analysis and Algebra, III (Proc. Conf., Kanpur, 1968), Academia, Prague, 1971, pp. 63-91. MR 44 \# 1616.

[Bo] N. Bourbaki, Eléments de mathématique. Livre III. Topologie générale. Chap. 1: Structures topologiques, 3ième éd., Actualités Sci. Indust., no. 1142, Hermann, Paris, 1961. MR 25 \#4480.

[Fe] P. Freyd, Abelian categories. An introduction to the theory of functors, Harper's Ser. in Modern Math., Harper \& Row, New York, 1964. MR 29 \#3517.

[Fr1] S. P. Franklin, A new metrization proof, General Topology and its Relations to Modern 
Analysis and Algebra, III (Proc. Conf., Kanpur, 1968), Academia, Prague, 1971, pp. 127-130. MR 44 \#987.

[Fr2] -, On epi-reflective hulls, Carnegie-Mellon Univ. Math. Dept. Research Report 70-23, 1970.

[Fr3] - Topics in categorical topology, Carnegie-Mellon University, Lecture Notes, 1970.

[F-T] S.P. Franklin and B.V.S. Thomas, $A$ categorical characterization of $\mathrm{CH}$, Carnegie-Mellon Univ. Math. Dept. Research Report 70-33, 1970.

[H] H. Herrlich, Topologische Reflexionen und Coreflexionen, Lecture Notes in Math., no. 78, Springer-Verlag, Berlin and New York, 1968. MR 41 \#988.

[H-S] H. Herrlich and G. E. Strecker, Algebra $\cap$ Topology = compactness, General Topology and Appl. 1 (1971), 283-287. MR 46 \# 7349.

[I] J. R. Isbell, Subobjects, adequacy, completeness and categories of algebras, Rozprawy Mat. 36 (1964). MR 29 \# 1238.

[M1] E. Michael, Bi-quotient maps and Cartesian products of quotient maps, Ann. Inst. Fourier (Grenoble) 18 (1968), fasc. 2, 287-302 (1969). MR 39 \#6277.

[M2] - On representing spaces as images of metrizable and related spaces, General Topology and Appl. 1 (1971), 329-343. MR 45 \#2681.

[Mo] K. Morita, Paracompactness and product spaces, Fund. Math. 50 (1961/62), 223-236. MR 24 \#A2365.

[N] N. Noble, Products with closed projections. II, Trans. Amer. Math. Soc. 160 (1971), 169-183. MR 44 \#979.

[P] V. I. Ponomarev, Axioms of countability and continuous mappings, Bull. Acad. Polon. Sci. Sér. Sci. Math. Astronom. Phys. 8 (1960), 127-134. (Russian) MR 22 \#7109.

[T] H. Tamano, On paracompactness, Pacific J. Math. 10 (1960), 1043-1047. MR 23 \# A2186.

[W] E. Wattel, The compactness operator in set theory and topology, Math. Centre Tracts, 21, Math. Centrum, Amsterdam, 1968. MR 39 \# 7551.

Department of Mathematics, Memphis State University, Memphis, Tennessee 38152 (Current address of S. P. Franklin and B.V.S. Thomas)

Department of Mathematics, University of Pittsburgh, Pittsburgh, Pennsylvania 15260

Current address (D. J. Lutzer): Department of Mathematics, Texas Tech University, Lubbock, Texas 79409 